\title{
Liver transplantation in Italy in the era of COVID 19: reorganizing critical care of recipients
}

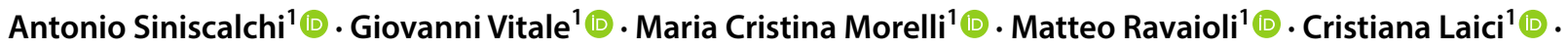

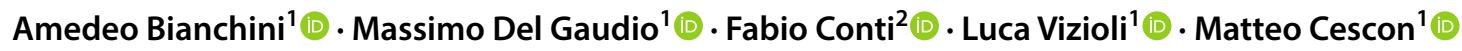

Received: 2 June 2020 / Accepted: 13 September 2020 / Published online: 26 September 2020

(c) Società Italiana di Medicina Interna (SIMI) 2020

\begin{abstract}
Transplant programs have been severely disrupted by the COVID-19 pandemic. Italy was one of the first countries with the highest number of deaths in the world due to SARS-CoV-2. Here we propose a management model for the reorganization of liver transplant (LT) activities and policies in a local intensive care unit (ICU) assigned to liver transplantation affected by restrictions on mobility and availability of donors and recipients as well as health personnel and beds. We describe the solutions implemented to continue transplantation activities throughout a given pandemic: management of donors and recipients' LT program, ICU rearrangement, healthcare personnel training and monitoring to minimize mortality rates of patients on the waiting list. Transplantation activities from February 22, 2020, the data of first known COVID-19 case in Italy's Emilia Romagna region to June 30, 2020, were compared with the corresponding period in 2019. During the 2020 study period, 38 LTs were performed, whereas 41 were performed in 2019. Patients transplanted during the COVID-19 pandemic had higher MELD and MELD-Na scores, cold ischaemia times, and hospitalization rates $(p<0.05)$; accordingly, they spent fewer days on the waitlist and had a lower prevalence of hepatocellular carcinoma $(p<0.05)$. No differences were found in the provenance area, additional MELD scores, age of donors and recipients, BMI, re-transplant rates, and posttransplant mortality. No transplanted patients contracted COVID-19, although five healthcare workers did. Ultimately, our policy allowed us to continue the ICU's operations by prioritizing patients hospitalized with higher MELD without any case of transplant infection due to COVID-19.
\end{abstract}

Keywords Liver transplant $\cdot$ Donors $\cdot$ Recipients $\cdot$ Healthcare personnel $\cdot$ Intensive care unit

\begin{tabular}{llll}
\multicolumn{2}{l}{ Abbreviations } & HCC & Hepatocellular carcinoma \\
BAL & Broncho-alveolar lavage & ICUs & Intensive care units \\
COVID-19 & Coronavirus disease 2019 & LT & Liver transplants \\
CT & Computed tomography & MELD & Mayo Clinic end-stage liver disease
\end{tabular}

Giovanni Vitale

giovanni.vitale@aosp.bo.it

Antonio Siniscalchi

antonio.siniscalchi@aosp.bo.it

Maria Cristina Morelli

mariacristina.morelli@aosp.bo.it

Matteo Ravaioli

matteo.ravaioli@aosp.bo.it

Cristiana Laici

cristiana.laici@aosp.bo.it

Amedeo Bianchini

amedeo.bianchini@aosp.bo.it

Massimo Del Gaudio

massimo.delgaudio@aosp.bo.it
Fabio Conti

fabio.conti2@studio.unibo.it

Luca Vizioli

luca.vizioli@aosp.bo.it

Matteo Cescon

matteo.cescon@aosp.bo.it

1 Dipartimento delle insufficienze d'organo e dei trapianti, Azienda Ospedaliero-Universitaria di Bologna, via Albertoni 15 , Bologna, Italy

2 Dipartimento di medicina interna, Ospedale degli Infermi di Faenza, AUSL Romagna, Faenza, Italy 


\section{NPS Naso-pharyngeal swab \\ PPE Personal protective equipment}

SARS-CoV-2 Severe acute respiratory syndrome coronavirus- 2

\section{Introduction}

Liver transplants (LTs) in the unprecedented era of the severe acute respiratory syndrome coronavirus-2 (SARS$\mathrm{CoV}-2)$ pandemic is of concern. The coronavirus disease 2019 (COVID-19) has crossed borders and infected approximately 10 million people worldwide although substantially more undiagnosed cases are likely [1]. The virus has proven difficult to contain partly due to its contagious nature and mild-to-nonexistent symptomatology in many cases. Nevertheless, the emergence of COVID-19 has impacted the transplants' community worldwide. Local and international societies for the study of LTs published several surveys and international position papers on the transplants' management during pandemic. Recommendations on COVID-19 screening of donors and recipients and transplant policies differed from country to country based on population infection rates and available diagnostic resources [2, 3]. In Italy, the first native case of COVID-19 was identified in Lombardy at the end of February 2020. All regions of the country have since registered infection from SARS-CoV-2. As of April 26 2020, there were 197,675 patients with COVID-19 in Italy, with 24,450 in the Emilia Romagna region and 4156 in its capital, Bologna [3]. Accordingly many of ICUs in the region have been reassigned to COVID-19 care. When Italy's lockdown ceased on May 18, cases in Emilia-Romagna had reached 27,$267 ; 5525$ of these cases were active.

While it is admirable to guarantee treatment for COVID19 subjects, on the others, it is essential to maintain a stringent standard of care in patients with potentially lethal diseases. Acute or chronic liver failure, is known, to have high mortality rate if left untreated conversely, appropriate treatment often results in longer survival. In recent years, critically ill LT candidates and recipients have benefitted from specialized critical transplant care; dedicated ICUs improved the outcome of patients with end-stage liver failure and attendant critical illnesses, and improved perioperative management during transplantation [4]. However, resources needed to perform LT successfully during a pandemic can be in short supply, and additional challenges such as exposure to the virus during immunosuppressive treatment, must be addressed.

In an era wherein the threat of COVID-19 will remain a reality for the foreseeable future, we must decide how to prioritize and treat non-COVID-19 populations that would otherwise survive if ICUs were operating at standard capacity $[2,5,6]$.
Transplant programs must assess the risk of donor transmission and the severity of disease in the recipient, and reduce the hazard for potential spread to healthcare workers [2]. This paper aims to provide data on the impact of COVID-19 on physicians and LT recipients in the setting of ICUs and to put forth possible clinical solutions and policies to mitigate the effects of the SARS-CoV-2 pandemic on LT patients and healthcare providers.

We discuss strategies such as donor screening, resource planning and management of LT recipient, all within in the context of a global pandemic. Moreover, we described routine surveillance of healthcare personnel and pragmatic reorganization of ICUs into the hospital by closed independent divisions to reduce the risk of contagion.

Lastly, transplantation activities from February 22, 2020, the date of the first recorded COVID-19 case in Emilia Romagna to June 30, 2020, were compared with the corresponding period in 2019.

\section{Methods and results}

\section{Donor management}

The blood of COVID-19-infected subjects carries nucleic acid of the virus in up to $15 \%$ of cases. To date, however, there is no evidence of COVID-19 transmission from the donor [7].

Nevertheless, given the higher sensitivity of alveolarbronchial lavage (BAL) compared to the nasopharyngeal swab (NPS), in the case of a donor, the Italian Transplant Authority (Centro Nazionale Trapianti) recommended carrying out the specific test for the presence of SARS-CoV-2 on respiratory secretions from BAL or possibly from deep broncho-aspirate, that is offered within $24-48 \mathrm{~h}$, prior to organ retrieval [8].

The central limit of BAL is the potential risk of healthcare workers contracting COVID-19 from the process of aerosolization. Since there is a risk of false negatives, especially in asymptomatic COVID-19 carriers, physicians are encouraged to review as much donor history as possible for fever, respiratory symptoms and radiographic findings [6]. In addition to performing a NPS in living donor LTs, some countries (e.g. Japan) recommend a 14-day isolation period before surgery [2]. Guidelines from the American Association for the Study of Liver Diseases recommend accepting only those grafts that have a low risk of delayed graft function, in order to reduce complications and post-operative hospitalization [6]. Donors who tested positive for COVID-19 were ineligible for organ donation. 


\section{Recipient management}

In Bologna University Hospital, all recipients underwent NPS before entering the operating room.

We ensured that backup transplant recipients were available at a location away from the Transplant Centre to reduce risk of a COVID-19 outbreak. For the same reason, potential transplant recipients entered the hospital at the latest possible time to minimize potential exposure to COVID-19 and family members were forbidden to enter the ward during the entire hospitalization period. However, it should be stressed that the sensitivity of the NPS for SARS-CoV-2 in asymptomatic subjects can be low, and negative test results do not exclude the possibility of a latent infection [6].

Thus, at the time of the donor's report on COVID-19 tests, recipients admitted for LT completed a standard online form to screen for symptoms of SARS-CoV-2 or recent exposure: fever, cough, shortness of breath, sore throat, diarrhoea, recent loss of sense of taste or smell contact with known COVID-19 carriers, and recent travel history. The decision-making process is summarised in Fig. 1.

The Centro Nazionale Trapianti also suggests administering the NPS for detecting SARS-CoV-2 in asymptomatic recipients awaiting organ transplantation from a living donor, within $72 \mathrm{~h}$ of the expected transplantation date. Using this method, the possibility of starting the test and therefore, response times, do not interfere with those of the transplant [8].

\section{Organization of liver transplants}

The increase of wait-list times was the leading risk of reduced organ recovery for COVID-19-related limitations on institutional resources and to the well-established threat of donor-derived disease transmission. Risk stratification is essential to prioritize the appropriate candidates for transplantation during the COVID-19 pandemic, including patients who score highly on the model for end-stage liver disease (MELD) scores, risk of decompensation, or tumour progression [6].

A multidisciplinary board composed of hepatologists, organ procurement teams, surgeons, critical care intensivists, microbiologists and nurses screened candidates, on a case-by-case basis considering the availability of resources such as ICU beds, ventilators, personal protective equipment (PPE) and supply of blood products in the LT process.

Since immunosuppression seems to play a protective role against SARS-CoV-2 infection and since there are no data on the course of COVID-19-infected LT, this condition should not constitute the primary limitation when deciding whether to perform the transplantation [7,9].

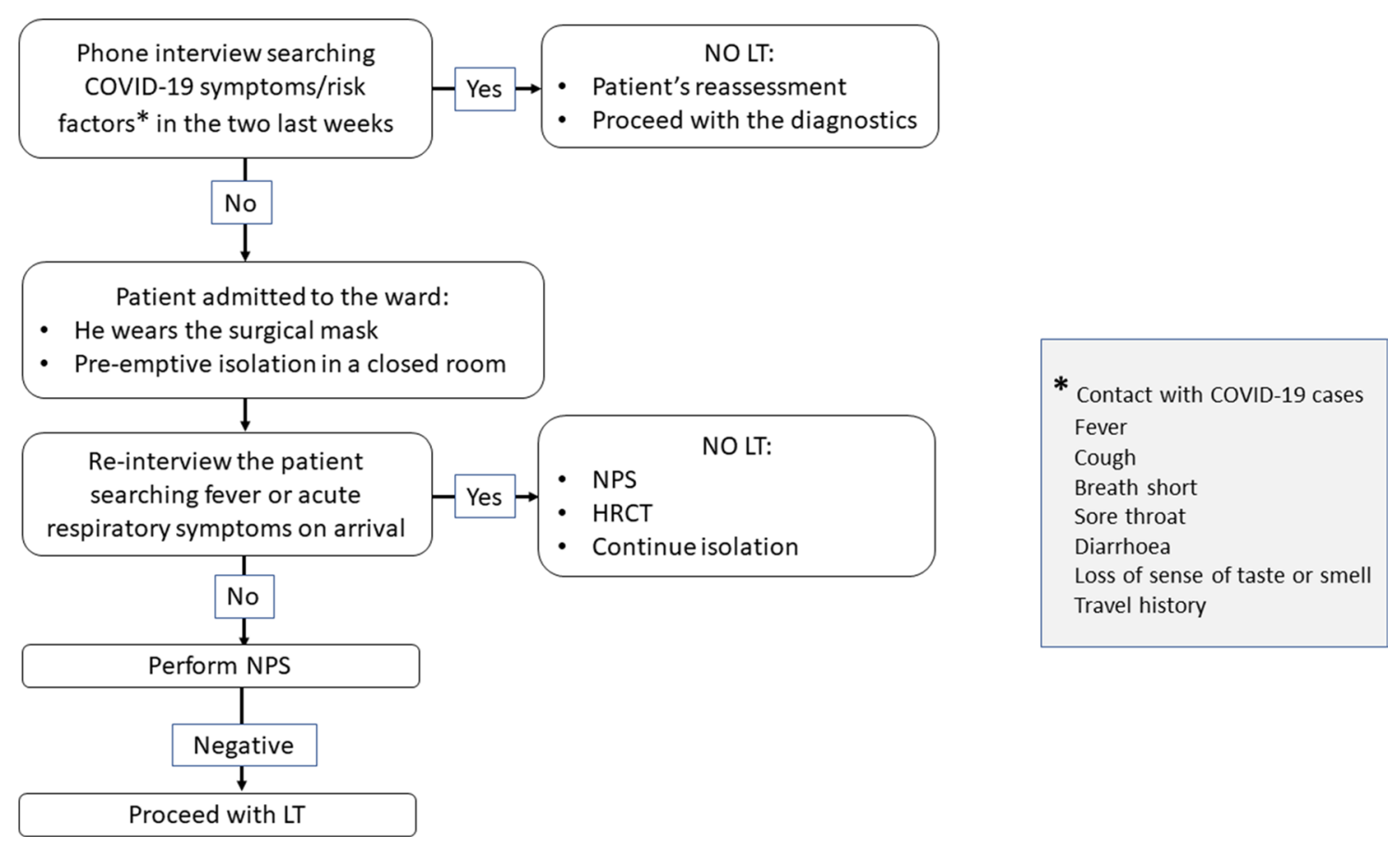

Fig. 1 Flowchart of recipient management. LT liver transplantation, NPS nasopharyngeal swab, HRTC high resolution computed tomography 


\section{LT ICU rearrangement}

Most countries were forced to plan for a substantial increase in critical care beds capacity. The main options were to add beds into a pre-existing ICU, including the provision of intensive care outside of ICUs, such as in high-dependency units, remodelled general wards, postanaesthesia care units, emergency departments, or deployable field units, and transfer of patients to designated hospitals and ICUs [10]. In the practical reallocation of human and material resources, in addition to the risk of the spread of viral SARS-CoV-2 infection, a current issue is the closure of non-COVID-19 ICUs such as ICUs specialized in transplant programs.

The ICU at the University Hospital in Bologna hosts the LT program and follows an average of 100 transplants in 2019 alone. The hospitalization capacity of this ICU, named the Abdominal Surgery and Liver Transplants ICU is eight beds. The course of treatment includes use of these beds, in addition to the use of semi-intensive and ordinary hospital beds according to a care intensity care logic. Although the eight beds are reserved for transplantation patients, they are located within an intensive area containing 12 additional beds (Fig. 2a). During the pandemic, many of the Hospital's ICU-beds were reallocated to patients with respiratory failure due to COVID-19. In the same building, 20 beds were implemented and allocated to COVID-19 patients, and eight beds to post-operative and post-transplant patients. To improve the safety of both patients and staff, we ensured physical separation of the two sectors and strictly monitored any cross-traffic. Beds devoted to LT-ICU have been obtained inside the spaces usually used in the operating room to guarantee complete isolation from the remaining ICU beds (Fig. 2b). On May 12, 2020, near the end of the lockdown in Italy, the placement of ICU's beds returned to their original arrangement. Faced with a pandemic that may pose additional challenges for transplant recipients, we only stratified patients with high MELD scores [11], hepatocellular carcinoma (HCC) or patients with clinically significant liver disease as well as recent liver decompensation or acute on chronic liver failure. Once selected, the candidate entered the General Surgery and Transplant Unit, already donned with PPE, and in any case, waited in a closed single room. Physicians, nurses and health personnel who approached the patient during work time wore PPE: they used N95 or surgical mask, gloves, disposable gown and visual protection according to the risk of the medical intervention. Relatives or acquaintances of the patient were forbidden from the ward. The patient underwent a NPS for COVID-19 and in the meantime, the patient was prepared for surgery as usual. Admission to the operating room occurred only once NPS results were negative; swab results were typically available within $4 \mathrm{~h}$. In the operating room, healthcare personnel wore N95 masks, covered by

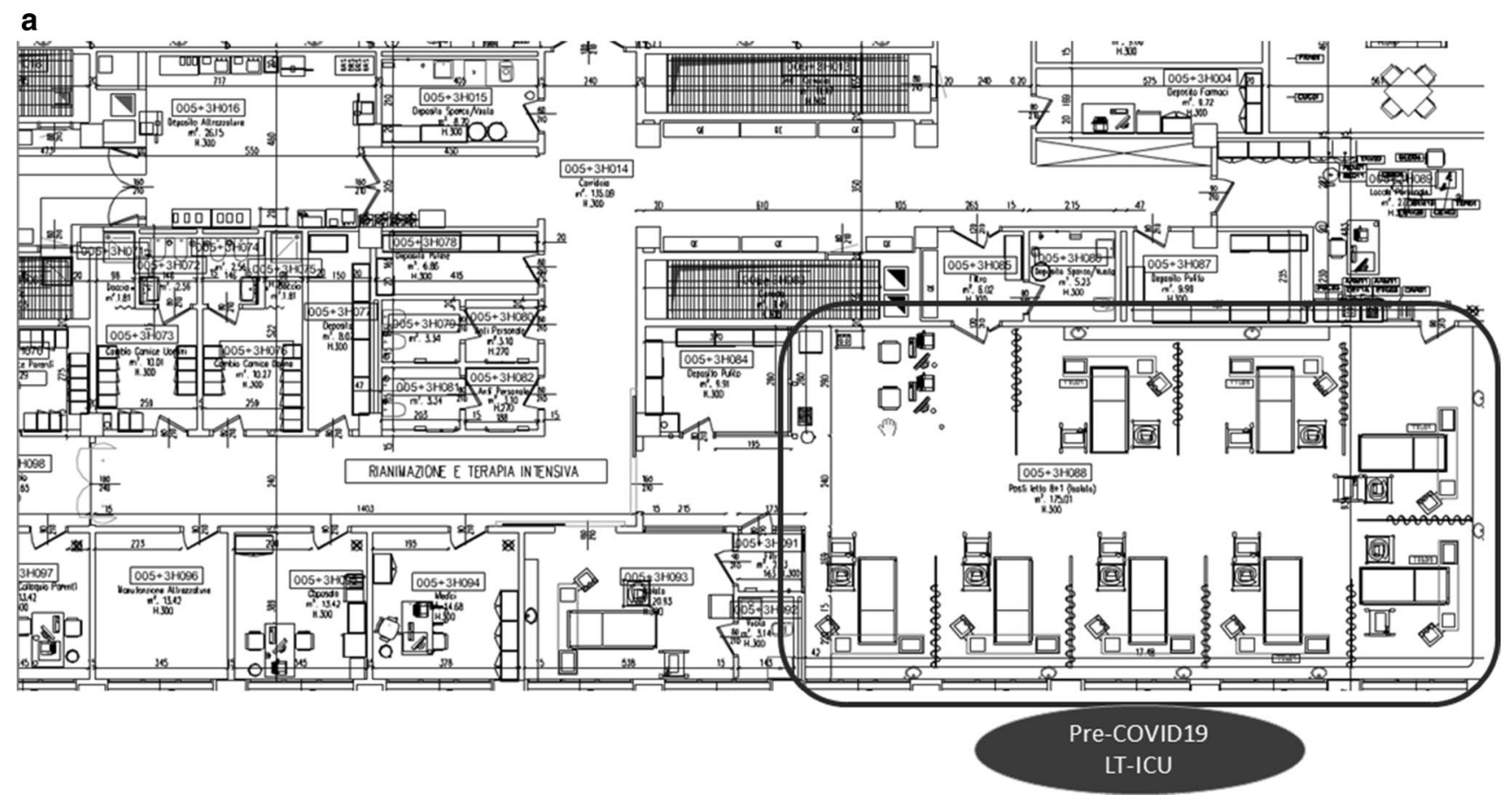

Fig. 2 a LT-Intensive care unit before arrangement for COVID-19: eight beds in an intensive area of 12 more beds. b LT-Intensive care unit rearrangement during COVID-19: eight beds to post-operative and post-transplant patients inside of the operating room 


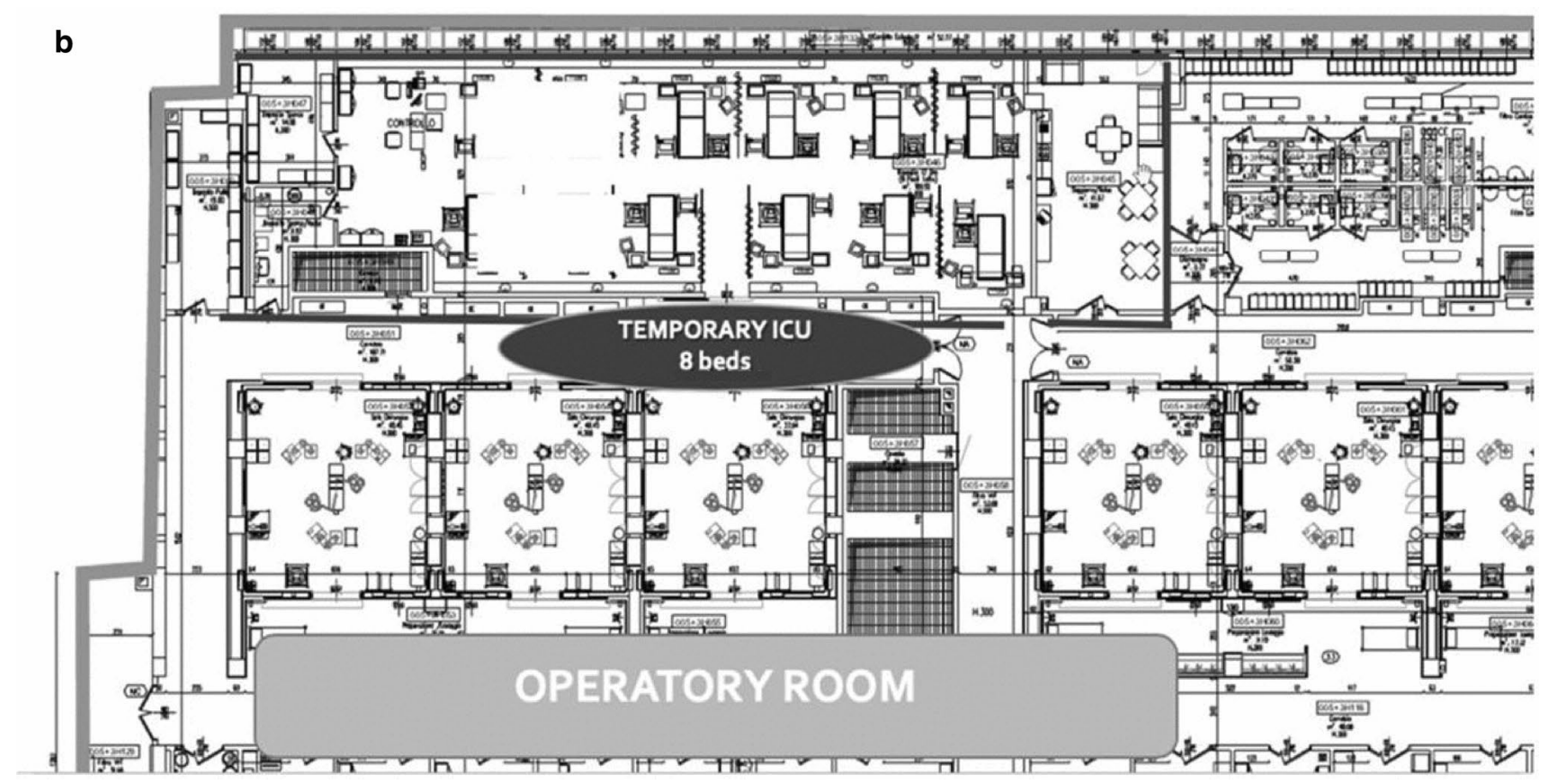

Fig. 2 (continued)

surgical mask, and visual protective gear. Following LT, the patient was transported to the ICU, where the medical and nursing staff who met the patient wore an N95 or surgical mask, a disposable gown, and visual protection. In addition to the usual graft monitoring, the post-operative follow up included several tests suggestive of COVID-19 infection: marker inflammatory agents including D-dimer, reactive protein $\mathrm{C}$, creatine phosphokinase, ferritin and interleukin-6. If there was clinical suspicion, we planned a BAL and lung computed tomography (CT) scan. Upon discharge we emphasized the post-transplant prevention measures: wash hands often, clean high-contact surfaces frequently, avoid large crowds and sick people. We also advised that patients avoid travel during the COVID-19 pandemic, to minimize potential exposure or spread. This was achieved by optimizing the use of telemedicine, and to consider the possibility of teleworking options. In the event of a fever or respiratory symptoms, patients were instructed to contact the Transplant Unit prior to visiting. The LT-ICU developed remotely administered screens to determine which symptomatic patients required evaluation, testing and management. Furthermore, all patients were educated on the broad spectrum of presentation of COVID-19 infection and on the need for more extended quarantine periods (up until two consecutive swabs are negative for COVID-19) due to the possibility of higher viral loads.

\section{Healthcare personnel training and monitoring}

On-site workers and other hospital staff are at higher risk of contracting COVID-19 [12]. Therefore, minimizing interactions between healthcare workers and patients, and minimizing the transport of patients within and between healthcare facilities, are critical to reducing the spread of SARS-CoV-2. Health care personnel receive continuous training on correct donning and doffing of PPE, including fit testing (e.g. 95 masks), precautions during the use of powered air-purifying respirators, and basic infection prevention principles, such as hand hygiene or surfaces disinfection [10].

Also, restricting health staff who are dedicated to LT to COVID-19-free departments with independent pathways can help reduce the risk of an outbreak. Every two weeks, all members of the transplant team were subjected to NPS to serology for $\operatorname{IgG}$ and IgM. For the determination of $\operatorname{IgM}$ and IgG antibodies against SARS-CoV-2 nucleocapsid protein and spike protein we used iFlash-SARS-CoV-2 (Shenzhen YHLO Biotech), a paramagnetic particle chemiluminescent immunoassay (CLIA). According to the manufacturer's inserts (V1.0 English Fd. 2020-02-20), the IgM and IgG cut-off was $10.0 \mathrm{kAU} / \mathrm{L}$. Classification accuracies exceeded 90\% (manufacturer threshold was $93 \%$ and $92.9 \%$ for sensitivity and specificity, respectively) [13]. Upon appearance of COVID-19 symptoms, staff self-isolated and implemented predefined backup plans to replace the quarantined 
Fig. 3 Screening and surveillance of healthcare personnel for COVID-19

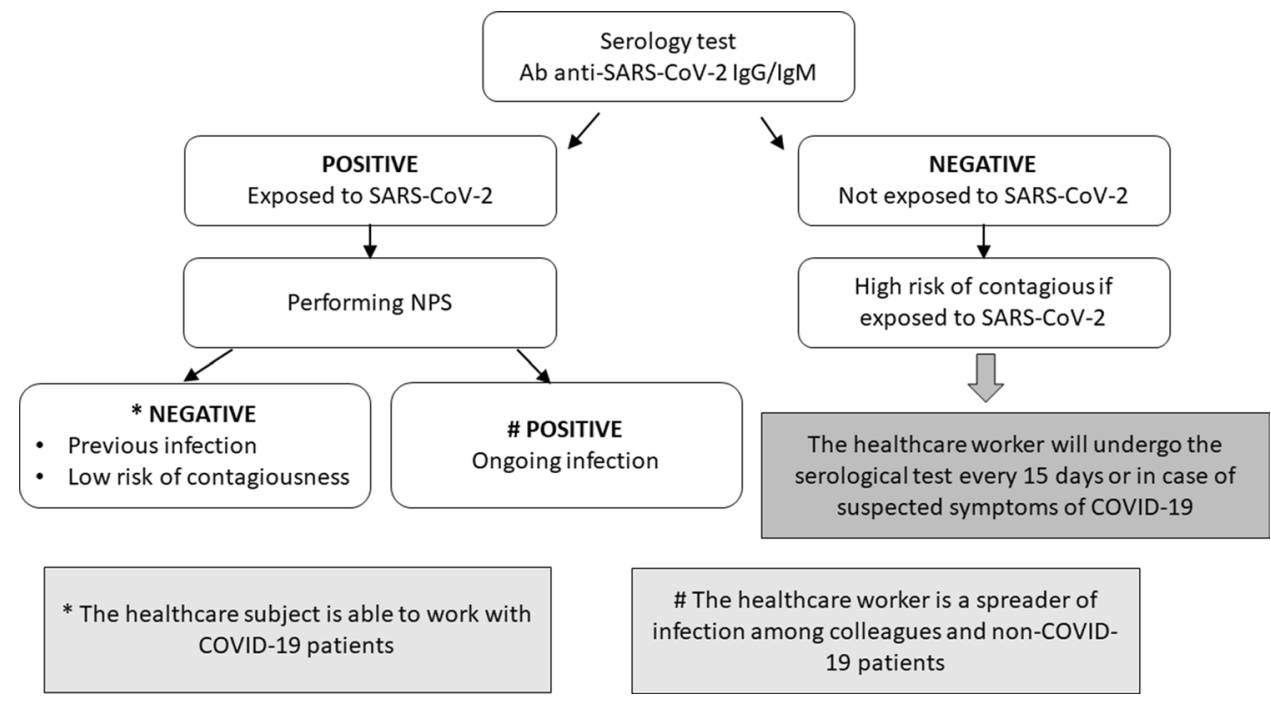

individual. Staff who assisted transplant patients avoided high-risk situations by maintaining transplant programs with closed parallel teams (without cross-team contact) to allow for continued transplant activity even in the case of a staff infection. Healthcare workers with SARS-CoV-2 can spread the virus readily; thus, they should be quarantined off-site until approved to return by local health authorities. Infected staff typically received two consecutive NPS every $24 \mathrm{~h}$ spaced 14 days apart from the positive result; readmittance to the workplace is permitted when both swabs are negative (Fig. 3).

The number of health workers infected with COVID-19 was low: one surgeon, one anaesthetist, two nurses and one nursing assistant were infected. No internal physician tested positive during the study period.

\section{Statistical analyses}

Categorical variables were expressed as number (\%), and quantitative variables as mean \pm standard deviation or as median (range). Chi-square or Fisher's exact test was used to compare categorical variables, while for quantitative variables, the Mann-Whitney $U$ test was used. The statistical software SPSS v.21.0 (SPSS 270 Inc., Chicago, IL, USA) was used for statistical analyses, and $p<0.05$ was considered significant for all tests.

\section{Liver transplants activities report}

As of February 22, 2020, the day of the first signalled COVID-19 case in the Emilia Romagna region, to June 30, 2020, the liver wait-list in Bologna registered 61 patients.
Throughout the same period in 2019, the wait-list registered 60 patients.

In the study period, four patients were suspended from the wait-list following a COVID-19-positive NPS; between them, only one patient required hospitalization for COVID19-related symptoms. One patient was transplanted once COVID-19 testing was negative.

To verify the impact of the COVID-19 pandemic on the activity of the Transplant Centre in Bologna, we analyzed the characteristics of the patients who received a LT from February 22 to June 30, 2020. Sex, age, type of liver disease, re-transplantation, body mass index, number of days on the transplant list, area of origin of recipients, MELD, MELD-Na, MELD-exceptions, mortality after LT, rates of hospitalization at the time of LT, cold ischemia, and the donor's age were considered. MELD-exceptions were determined according to national and regional waiting list prioritization policies. The etiology of liver disease was divided into cirrhosis, HCC, "others", and re-transplants, while the area of residence was categorized as either Emilia-Romagna, Northern Italy, or elsewhere in Italy. Only in a single case was the liver allocated to another centre for a logistical reason: no ICU bed was available for transplantation. A total of 38 LTs were performed in COVID-era study period, compared to 41 during the same period in 2019.

No significant differences were found between the 2020 and 2019 study period with respect to age $(54.8 \pm 10.2$ vs $57.5 \pm 10.4$ years; $p=0.171)$, BMI $(26.6 \pm 6.29$ vs $26.8 \pm 5.1$; $p=717)$, rates of re-transplantation $[1(2.6 \%)$ vs $3(7.3 \%)$; $p=0.667$, donor's age (59.7 years \pm 19.3 vs $60.2 \pm 16.2$; $p=0.998)$ and MELD exceptions [19 (50\%) vs $31(75 \%)$; $p=0.865]$. Notably, there were no differences in the zone of residence of recipients despite the lockdown and travel difficulties: 12 patients in 2019 come from a region different from Emilia Romagna and North of Italy while resulted in 
Table 1 Features of recipients, donors and transplants performed in Bologna University Hospital

\begin{tabular}{|c|c|c|c|}
\hline Patients & $\begin{array}{l}\text { LT in } \\
\text { COVID era } \\
(n=38)\end{array}$ & $\begin{array}{l}\mathrm{LT} \text { in } 2019 \\
\text { period }^{\mathrm{b}} \\
(n=41)\end{array}$ & $p$ \\
\hline Males $(n / \%)$ & $23(60.5)$ & $34(82.9)$ & 0.043 \\
\hline $\begin{array}{l}\text { Age at time of LT (years, } \\
\text { mean } \pm S D \text { ) }\end{array}$ & $54.8 \pm 10.2$ & $57.5 \pm 10.4$ & 0.171 \\
\hline $\begin{array}{l}\text { Days in waiting list (median, } \\
\text { range) }\end{array}$ & $128(1-1240)$ & $243(1-1347)$ & 0.042 \\
\hline \multicolumn{4}{|l|}{ Residence $(n / \%)$} \\
\hline ER & $24(63.1)$ & $22(53.6)$ & 0.494 \\
\hline North Italy & $2(5.3)$ & $2(4.9)$ & 1 \\
\hline Rest of Italy & $12(31.6)$ & $17(41.5)$ & 0.484 \\
\hline \multicolumn{4}{|l|}{ Aetiology $(n / \%)$} \\
\hline $\mathrm{HCC}$ & $12(31.6)$ & $27(65.8)$ & 0.003 \\
\hline Cirrhosis & $18(47.4)$ & $10(24.4)$ & $\mathbf{0 . 0 3 8}$ \\
\hline Re-LTs & $3(7.9)$ & $2(4.9)$ & 0.667 \\
\hline Others & $5(13.1)$ & $2(4.9)$ & 0.252 \\
\hline Exceptions to MELD $(n / \%)$ & $19(50)$ & $31(75)$ & 0.865 \\
\hline $\mathrm{BMI}($ mean $\pm \mathrm{SD})$ & $26.6 \pm 6.29$ & $26.8 \pm 5.1$ & 0.717 \\
\hline $\operatorname{MELD}($ mean \pm SD $)$ & $20.4 \pm 9.3$ & $15.6 \pm 9.6$ & 0.008 \\
\hline MELD-Na $($ mean $\pm \mathrm{SD})$ & $22.5 \pm 8.6$ & $17.2 \pm 9.6$ & 0.016 \\
\hline $\begin{array}{l}\text { Donor's age (years, } \\
\text { mean } \pm \mathrm{SD} \text { ) }\end{array}$ & $59.7 \pm 19.3$ & $60.2 \pm 16.2$ & 0.988 \\
\hline $\begin{array}{l}\text { Cold ischaemia (min, } \\
\text { mean } \pm \mathrm{SD})\end{array}$ & $474 \pm 124$ & $413 \pm 120$ & 0.005 \\
\hline \multicolumn{4}{|l|}{ Hospitalization at LT $(n / \%)$} \\
\hline No & $18(47.4)$ & $29(70.7)$ & 0.041 \\
\hline In Ward & $19(50)$ & $10(24.4)$ & 0.022 \\
\hline In ICU & $1(2.6)$ & $2(4.9)$ & 1 \\
\hline Death $(n / \%)$ & $1(2.6)$ & $3(7.9)$ & 0.618 \\
\hline
\end{tabular}

Chi-square, Fisher-test and Mann-Whitney were used appropriately to compare categorical and continuous variables, respectively

BMI body mass index, COVID-19 coronavirus disease 19, ER Emilia Romagna, $H C C$ hepatocellular carcinoma, ICU Intensive Care Unit, $L T$ liver transplantation, $m$ minutes, $M E L D$ - $N a$, Mayo-end-stage liver disease-sodium, $n$ number, $r e L T$ re-liver transplantation, $S D$ standard deviation

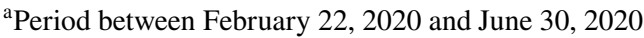

${ }^{\mathrm{b}}$ Period between February 22, 2019 and June 30, 2019

17 the previous year [12 (31.6\%) vs $17(41.5 \%) ; p=0.484]$. During the COVID-19 pandemic, more critical patients were transplanted than in 2019: MELD and MELD-Na scores were higher $(20.4 \pm 9.3$ and $22.5 \pm 8.6$ vs $15.6 \pm 9.6$ and 17.2 $\pm 9.6 ; p=0.008$ and $p=0.016$, respectively). More patients were hospitalized in a ward at the time of the LT [ 19 $(50 \%)$ vs $10(24.4 \%) ; p=0022]$, liver failure was more common than HCC [18 (47.4\%) liver cirrhosis cases in 2020 vs $10(24.4 \%)$ in $2019 ; p=0.038)$ and days spent on the waiting list were fewer [128 (1-1240) in 2020 vs $243(1-1347)$ in 2019; $p=0.042$ ). Relevant characteristics of the recipients, the donors, and the LT are summarized in Table 1. The transplants were performed in critically ill patients likely to die without surgery. Notably, cold ischemic time was on average higher in the 2020 study period relative to the same period in $2019(474 \pm 124$ vs $413 \pm 124 \mathrm{~min} ; p=0.005)$, which can be explained by the additional time required to obtain the swab report. No significant differences were observed in post-transplant mortality rates in the two periods studied [ $1(2.6 \%)$ in 2020 vs $3(7.9 \%)$ in $2019 ; p=0.618$ ]. To date, COVID-19 has not been confirmed in our LT patients.

\section{Conclusions}

The low availability of beds in COVID-19-free ICUs depends on the fast overstaying beds in COVID-19 ICUs due to the prolonged intubation required by many of these patients (often 15-20 days of mechanical ventilation) [14]. Thus, the complex decision-making process involved in whether to proceed with LT has been rendered significantly more challenging by the ongoing COVID-19 outbreak. During the pandemic, we have had to continue monitor our resources and any implications for patients waiting for a LT. We opted not to discontinue the LT program given the reduction in organ recovery due to the limitations on institutional resources of COVID-19, and this is expected to significantly increase waiting times to receive a LT. The dilemma is to assess the risks and rewards of waitlisted patients who require a transplant to resolve their primary disease but who are also at risk of severe infections, with possibly fatal outcomes. However, there are no data suggesting that the immunosuppression worsens the course of COVID-19; indeed, it may have a protective role against the cascades of inflammatory cytokines activated by the virus [7, 9]. Furthermore, employees in the ICU and the transplant ward can contract asymptomatic COVID-19 by spreading the infection both within and beyond the hospital confines. With these considerations as a starting point, we have maintained a dedicated path for LT during the COVID19 outbreak, with some limitations. We took a careful risk stratification approach and chose to transplant waitlist candidates with high MELD scores or HCC based on the risk of disease progression, excluding positive COVID-19 transplant candidates.

Data on LT transplants in the COVID-19 era of 2020, compared with the same period in 2019 , confirmed the tendency of the Transplant Centre in Bologna to transplant more critical patients, as suggested by higher scores of liver failure (on the MELD and MELD-Na) and a higher rate of hospitalization of LT recipients in 2020 and shorter time spent on the waiting list. The main measures we were required to introduce in our standard of care were the exclusion of COVID-19-positive donors and recipients, staff 
training, and the application of infection control measures to minimize the risk of spread.

Despite the difficulties related to the COVID-19 pandemic, and the greater severity of illness in these patients, the mortality rates of transplants and re-transplantation rates remained low and did not differ significantly from those of the previous year (Table 1).

At present, there are no reliable data on the impact that an infection derived from a COVID-19 donor could have on the medical system and the broader community of recipients [5].

The acquisition of knowledge about SARS-CoV-2 is accumulating rapidly. There are many unknown factors of relevance to the community in general and the transplant community, particularly in Italy. When the West Nile virus emerged in the United States in 1999, we quickly learned that transplant patients were at particular risk for significant morbidity and mortality from blood and organ transfusions obtained from donors with asymptomatic infection [15]. The optimal approach to donor screening can change over time as more data accumulates. We encourage the screening of all donors for possible COVID-19 exposure and clinical symptoms. Considering that samples should be tested for sufficient quantities of replicating virus and considering optimal collection site procedures, we invariably recommend collecting lower respiratory samples for COVID-19. BAL is a more sensitive real-time PCR-based methods of NPS, which can be $\sim 30 \%$ negative in the early stage of the disease because the SARS-CoV-2 viral load may be low: BAL fluid specimens achieve in up to $93 \%$ using real-time PCR-based methods [16].

One of the biggest questions we have encountered is how best to maintain patients with acute on chronic liver failure in intensive care awaiting transplantation. SARS-CoV-2 is a single positive-stranded RNA virus that replicates using a viral-encoded RNA-dependent RNA polymerase, and it binds and is internalized in the target cells through the angiotensin- 2 converting enzyme, which acts as a functional receptor [17]. The angiotensin- 2 converting enzyme is present in biliary and hepatic epithelial cells; therefore, the liver is a potential target of infection. In the event of infection, it will be difficult to distinguish whether the elevated liver biochemistry values are due to the SARS-CoV-2 infection itself or to the worsening liver disease. Complications from COVID-19, including myositis (especially with aspartate transaminase $\rightarrow$ alanine transaminase), cytokine release syndrome, and ischemia/hypotension, are also common in liver disease [17]. Routine labs that include a complete blood count and a comprehensive metabolic panel can provide useful indirect evidence of infection. Inflammatory markers, including D-dimer, reactive protein $\mathrm{C}$, creatine phosphokinase, and ferritin, are often elevated in patients affected with COVID-19. The chest ultrasound is now daily practice in the diagnosis of COVID-19 pneumonia, whereas lung CT scans are reserved for dubious cases because it is more sensitive than ultrasounds.

Healthcare facility transmissions of COVID-19 have occurred; given the potential for increased infectivity, anyone suspected of SARS-CoV-2 must follow strict isolation precautions. Although the virus is not airborne, we recommend the use of airborne precautions and PPE. We have minimized interactions between healthcare professionals and between healthcare professionals and patients, decreased the transport of patients within and between independent healthcare facilities using a parallel path and dedicated exclusive medical teams for patients hospitalized with COVID-19.

We minimized the presence in the department of nonessential team members as well as students, observers and research staff to minimize the risk of exposure. Also, all asymptomatic health professionals involved in patient care are currently subjected to periodic blood screening for IgM-IgG antibodies against SARS-CoV-2. Although five health workers have contracted the virus, no transplanted patient was infected, as a result of the surveillance programs and safety measures implemented.

However, it is imperative to develop new, cost-effective and reliable serological diagnostics. Early diagnosis helps mitigate the spread of the disease, which remains the main problem in an ICU hosting liver transplanted or transplant candidates. If there is a second wave of COVID-19 or if another pandemic occurs, we have acquired the necessary expertise to ensure a sustainable transplant activity. As long as there is only one case, we have to compare how we were in full outbreak to ensure the continuation of standard LT programs in the setting of intensive care.

Funding This manuscript has not been funded.

\section{Compliance with ethical standards}

Conflict of interest The authors have no relevant affiliations or financial involvement with any organization or entity with a financial interest in or financial conflict with the subject matter or materials discussed in the manuscript.

Ethical approval All procedures performed in studies involving human participants were in accordance with the ethical standards of the institutional and/or national research committee and with the 1964 Helsinki declaration and its later amendments or comparable ethical standards.

Informed consent Informed consent was obtained from patients before liver transplantation. 


\section{References}

1. World Health Organization (2020) Coronavirus disease (COVID19) Pandemic. https://www.who.int/emergencies/diseases/novel -coronavirus-2019. Accessed 30 June 2020

2. Kumar D, Manuel O, Natori Y, Egawa H, Grossi P, Han S et al (2020) COVID-19: a global transplant perspective on successfully navigating a pandemic. Am J Transplant. https://doi.org/10.1111/ ajt.15876

3. Dipartimento della Protezione Civile (2020) Emergenza Coronavirus. https://www.protezionecivile.gov.it/attivita-rischi/risch io-sanitario/emergenze/coronavirus. Accessed 18 May 2020

4. Niemann CU, Kramer C (2011) Transplant critical care: standards for intensive care of the patient with liver failure before, and after transplantation. Liver Transplant 17(5):485-487. https://doi. org/10.1002/lt.22289

5. Ahn C, Amer H, Anglicheau D, Ascher N, Baan C, Bat-Ireedui $\mathrm{B}$, Berney $\mathrm{T}$ et al (2020) Global transplantation COVID report March 2020. Transplantation. https://doi.org/10.1097/TP.00000 00000003258 (Epub ahead of print)

6. Fix OK, Hameed B, Fontana RJ, Kwok RM, McGuire BM, Mulligan DC et al (2020) Clinical best practice advice for hepatology and liver transplant providers during the COVID-19 pandemic: AASLD Expert Panel consensus statement. Hepatology. https:// doi.org/10.1002/hep.31281(Epub ahead of print)

7. Huang C, Wang Y, Li X, Ren L, Zhao J, Hu Y et al (2020) Clinical features of patients infected with 2019 novel coronavirus in Wuhan, China. Lancet 395(10223):497-506. https://doi. org/10.1016/S0140-6736(20)30183-5

8. Ministero della Salute (2020) Centro Nazionale Trapianti. Comunicati e notizie. https://www.trapianti.salute.gov.it/trapianti/archi vioComunicatiNotizieCnt.jsp. Accessed 26 Apr 2020

9. D'Antiga L (2020) Coronaviruses and immunosuppressed patients. The facts during the third epidemic. Liver Transplant. https://doi. org/10.1002/lt.25756(Epub ahead of print)
10. Phua J, Weng L, Ling L, Egi M, Lim C, Divatia J et al (2020) Intensive care management of coronavirus disease 2019 (COVID19): challenges and recommendations. Lancet Respir Med. https:// doi.org/10.1016/S2213-2600(20)30161-2(Epub ahead of print)

11. Wiesner R, Edwards E, Freeman R, Harper A, Kim R, Kamath P et al (2003) Model for end-stage liver disease (MELD) and allocation of donor livers. Gastroenterology 124(1):91-96

12. Remuzzi A, Remuzzi G (2020) COVID-19 and Italy: what next? Lancet 395(10231):1225-1228. https://doi.org/10.1016/S0140 $-6736(20) 30627-9$ (Epub ahead of print)

13. Plebani M, Padoan A, Negrini D, Carpinteri B, Sciacovellia L (2020) Diagnostic performances and thresholds: the key to harmonization in serological SARS-CoV-2 assays? Clin Chim Acta 509:1-7

14. Rosenbaum L (2020) Facing Covid-19 in Italy_ethics, logistics, and therapeutics on the epidemic's front line. N Engl J Med. https ://doi.org/10.1056/NEJMp2005492(Epub ahead of print)

15. Winston D, Vikram H, Rabe I, Dhillon G, Mulligan D, Hong $\mathrm{J}$ et al (2014) Donor-derived West Nile Virus infection in solid organ transplant recipients: report of four additional cases and review of clinical, diagnostic, and therapeutic features. Transplantation 97(9):881-889

16. Wang W, Xu Y, Gao R, Lu R, Han K, Wu G et al (2020) Detection of SARS-CoV-2 in different types of clinical specimens. JAMA. https://doi.org/10.1001/jama.2020.3786(Epub ahead of print)

17. Sun J, Aghemo A, Forner A, Valenti L (2020) COVID-19 and liver disease. Liver Int. https://doi.org/10.1111/liv.14470 (Epub ahead of print)

Publisher's Note Springer Nature remains neutral with regard to jurisdictional claims in published maps and institutional affiliations. 